7. van Adrichem EJ, Meulenbroek RL, Plukker JT, Groen H, van Weert E. Comparison of two preoperative inspiratory muscle training programs to prevent pulmonary complications in patients undergoing esophagectomy: a randomized controlled pilot study. Ann Surg Oncol. 2014;21:2353-60.

8. Licker M, Karenovics W, Diaper J, Fresard I, Triponez F, Ellenberger C, et al. Short-term preoperative high-intensity interval training in patients awaiting lung cancer surgery: a randomized controlled trial. J Thorac Oncol. 2017;12:323-33.

https://doi.org/10.1016/j.jtcvs.2018.09.076

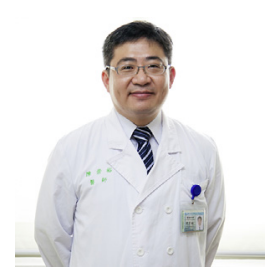

\section{INSPIRATORY MUSCLE TRAINING FOR REDUCING POSTOPERATIVE PULMONARY COMPLICATIONS: A SIMPLE METHOD BUT}

\section{SHORT OF DATA?}

\section{Reply to the Editor:}

In a letter to the Editor, Licker and Diaper ${ }^{1}$ expressed concern about the compliance and withdrawal of inspiratory muscle training (IMT) protocol and inconsistent inclusion criteria in the meta-analysis made by Ge and colleagues. ${ }^{2}$ After the exclusion of 5 studies mentioned by Licker and Diaper, ${ }^{1}$ only 536 patients (originally 784) can be analyzed in the study. That may get a different result.

A weak point of the meta-analysis is that although 13 trials were included in the study, there were only 372 patients receiving IMT and 369 patients in the control group. In addition, there were different IMT protocols in every trial. The efficacy of IMT for preventing postoperative pulmonary complications (PPCs) is also confounded by other factors that may prevent PPCs, such as smoking cessation and correcting anemia. ${ }^{3}$ These trials may indicate that IMT is one but not the only method of preventing PPCs. In the absence of confirmation by a large, randomized controlled trial, the efficacy of IMT in PPC prevention remains doubtful. We appreciate the viewpoint of Licker and Diaper, ${ }^{1}$ and we hope that Ge and colleagues will clarify the study result. The accompanying editorial commentary ${ }^{4}$ also points out that IMT is time-consuming and needs staff time. So, is IMT a simple method? Until we have standardize IMT's protocol and conducted large clinical trials with more patients, the method remains difficult.

\section{Chien-Hong Chou, MD \\ Chung-Yu Chen, MD, PhD \\ Department of Internal Medicine \\ National Taiwan University Hospital Yun-Lin Branch Yun-Lin County, Taiwan}

\section{References}

1. Licker M, Diaper J. Clinical impact of preoperative inspiratory muscle training: we need further studies! J Thorac Cardiovasc Surg. 2019;157:175-6.

2. Ge X, Wang W, Hou L, Yang K, Fa X. Inspiratory muscle training is associated with decreased postoperative pulmonary complications: evidence from randomized trials. J Thorac Cardiovasc Surg. 2018;156:1290-300.e1295.
Authors have nothing to disclose with regard to commercial support.

3. Miskovic A, Lumb AB. Postoperative pulmonary complications. Br J Anaesth. 2017:118:317-34.

4. Chou $\mathrm{CH}$, Chen CY. Inspiratory muscle training for reducing postoperative pulmonary complications: a simple method that we hope is effective. J Thorac Cardiovasc Surg. 2018;156:1301-2.

https://doi.org/10.1016/j.jtcvs.2018.10.053

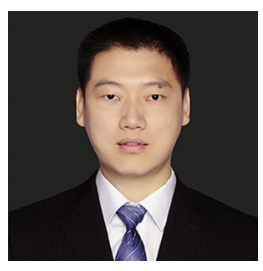

REDUCING
POSTOPERATIVE
PULMONARY
COMPLICATIONS:
STILL A LONG WAY
TO GO
Reply to the Editor:

We thank Licker and Diaper for their interest in our article. ${ }^{1}$ Postoperative pulmonary complications (PPCs) are one of the most frequent causes of postoperative morbidity and mortality. The purpose of inspiratory muscle training (IMT) is to improve inspiratory muscle strength and endurance. No powered trials, however, can confirm that IMT can decrease the incidence of PPCs. Evidence from randomized, controlled trials was insufficient to reach definitive conclusions. Our team applied the statistical method of trial sequential analysis to complete the metaanalysis and found that preoperative IMT is associated with improved maximum inspiratory pressure and decreased PPCs in patients undergoing cardiothoracic or upper abdominal surgery.

In terms of confounding factors, Licker put forward very good questions about the factors that may contribute to the success of the intervention and the inclusion criteria. We agree that these concerns may limit widespread application and clinical effectiveness. As Chou and $\mathrm{Chen}^{2}$ said in their editorial commentary, getting information from original clinical trials is a hallmark of meta-analysis, but sometimes it is not so easy. We sent email to all corresponding authors of included studies for the missing data. The study of Heynen and colleagues ${ }^{3}$ can be found in the Diseases of the Esophagus Wiley Online Library (https://onlinelibrary. wiley.com/journal/14422050), but the corresponding author's contact information did not show. One study was a nonrandomized trial, but it was a double-blind pilot study, and we evaluated the quality through the Cochrane risk of bias tool. ${ }^{4}$ The intervention in 2 studies was preoperative and postoperative IMT, which may also have had an impact on our current results. 5,6 The study by van Adrichem and colleagues ${ }^{7}$ included 2 intervention groups (a high-intensity group and an endurance group), rather 
Authors have nothing to disclose with regard to commercial support.

than usual care. The result of IMT associated with decreased PPCs suggests that the effect of IMT on improving pulmonary outcomes may be even stronger than our analysis suggests. ${ }^{1}$ Because of the unclear proportions of laparoscopic or thoracoscopic versus open operations, whether minimally invasive surgery would eliminate the observed effect that patients who had IMT had significantly fewer PPCs after open surgery but not after thoracoscopy or laparoscopy is not certain. These limitations were illustrated in our section on strengths and limitations. Preoperative smoking cessation, correcting preoperative severe anemia, avoidance of general anesthesia in favor of regional anesthesia, protective ventilation, correction of anemia, and improvement of nutritional status all have an effect on PPC prevention. ${ }^{8,9}$ We need to find a higher level of evidence to identify which method is the best way to guide clinical practice.

In conclusion, to meet a higher evidence level and to determine the appropriate surgical candidates and the modalities of physical training when patients undergoing cardiothoracic or upper abdomen surgery, more doubleblind and unbiased randomized, controlled trials are necessary.

Xiao-Qing Ge, $M D^{a}$
Wen-Jie Wang, $M D^{b}$
Kun-Peng Yang, MD, PhD ${ }^{a}$
Qing-Quan Luo, MD, PhD
${ }^{c}$ Department of Thoracic Surgery
The Second Affiliated Hospital of Zhengzhou University
Zhengzhou, China
${ }^{b}$ Second Clinical Medical College of Lanzhou University
Lanzhou, China
${ }^{c}$ Department of Oncology
Shanghai Chest Hospital
Shanghai Jiao Tong University
Shanghai, China

\section{References}

1. Ge X, Wang W, Hou L, Yang K, Fa X. Inspiratory muscle training is associated with decreased postoperative pulmonary complications: evidence from randomized trials. J Thorac Cardiovasc Surg. 2018;156:1290-300.e5.

2. Chou $\mathrm{CH}$, Chen $\mathrm{CY}$. Inspiratory muscle training for reducing postoperative pulmonary complications: a simple method that we hope is effective. $J$ Thorac Cardiovasc Surg. 2018;156:1301-2.

3. Heynen H, de Jonge C, Kerkkamp H, Willms J, Sosef M. Preconditioning in patients undergoing esophagectomy: a randomized controlled pilot study. Dis Esophagus. 2012;25:84A.

4. Dettling DS, van der Schaaf M, Blom RL, Nollet F, Busch OR, van berge Henegouwen MI. Feasibility and effectiveness of pre-operative inspiratory muscle training in patients undergoing oesophagectomy: a pilot study. Physiother Res Int. 2013;18:16-26.
5. Savci S, Degirmenci B, Saglam M, Arikan H, Inal-Ince D, Turan HN, et al Short-term effects of inspiratory muscle training in coronary artery bypass graft surgery: a randomized controlled trial. Scand Cardiovasc J. 2011;45:286-93.

6. Brocki BC, Andreasen JJ, Langer D, Souza DS, Westerdahl E. Postoperative inspiratory muscle training in addition to breathing exercises and early mobilization improves oxygenation in high-risk patients after lung cancer surgery: a randomized controlled trial. Eur J Cardiothorac Surg. 2016;49:1483-91.

7. van Adrichem EJ, Meulenbroek RL, Plukker JT, Groen H, van Weert E. Comparison of two preoperative inspiratory muscle training programs to prevent pulmonary complications in patients undergoing esophagectomy: a randomized controlled pilot study. Ann Surg Oncol. 2014;21:2353-60.

8. Miskovic A, Lumb AB. Postoperative pulmonary complications. Br J Anaesth 2017;118:317-34.

9. Licker M, Karenovics W, Diaper J, Frésard I, Triponez F, Ellenberger C, et al. Short-term preoperative high-intensity interval training in patients awaiting lung cancer surgery: a randomized controlled trial. J Thorac Oncol. 2017;12:323-33.

https://doi.org/10.1016/j.jtcvs.2018.10.067

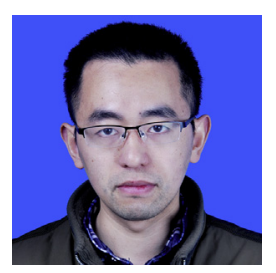

THE BENEFIT OF

RECRUITMENT

MANEUVER DURING

NONINVASIVE

VENTILATION IN

PATIENTS

AFTER CARDIAC

\section{SURGERY REMAINS UNCLEAR}

\section{To the Editor:}

In their recent Journal article, Miura and colleagues ${ }^{1}$ reported that in patients undergoing cardiac surgery with a ratio of $\mathrm{PaO}_{2}$ to fraction of inspired oxygen less than 300 and a radiographic atelectasis score (RAS) of at least 2, the use of noninvasive ventilation with recruitment maneuver is associated with improved oxygenation, improved RAS, and fewer days of oxygen supplementation during the hospital stay.

Several limitations, however, should be clarified. One important issue is that the blinding of patients and the intensive care unit team cannot be guaranteed in the study of Miura and colleagues. ${ }^{1}$ Although the comparison of final $\mathrm{PaO}_{2}$ at room air between the 2 groups was significant, the absolute difference of $\mathrm{PaO}_{2}$ was small $(67.2 \pm 5.9$ vs $73.7 \pm 7.1 \mathrm{~mm} \mathrm{Hg} ; P=.007)$. Because the value of $\mathrm{PaO}_{2}$ changes continuously during the whole treatment, the selection of $\mathrm{PaO}_{2}$ in each patient could easily be biased if the investigators were not blinded. Actually, we believe that blinding bias already existed, as we noticed the use of oxygen at intensive care unit discharge was more frequently in the control group $(14 / 16$ vs $4 / 18 ; P<.001)$, despite the small difference in $\mathrm{PaO}_{2}(6.5 \mathrm{~mm} \mathrm{Hg})$. Second, Figure 2 of the article of Miura and colleagues ${ }^{1}$ shows that $94.4 \%$ of patients in the recruitment group changed to an RAS of 0 , versus $12.5 \%$ of patients in the control group $(P<.001)$. The evaluation was performed by physicians blinded to the group allocation. According the definition of RAS, however, RAS of 0 to 1 ( 0 without atelectasis, 1 line of atelectasis or discrete infiltration) indicates only 